\title{
Influence de la pénicilline sur la qualité d'un fromage de type Gruyère fait à partir de lait de brebis
}

par

Emmanuel ANIFANTAKIS*

\section{INTRODUCTION}

Il est bien connu que la présence d'antibiotique dans le lait pose de graves problèmes aux usines de fabrication de fromages $[1,8]$.

Dans notre précédente étude [2], nous avons étudié l'influence de la pénicilline sur la qualité du fromage Feta et il a été constaté que par la présence de plus de $0,1 \mathrm{u} . \mathrm{i} / \mathrm{ml}$ d'antibiotique, dans le lait de fabrication, la qualité des fromages était sensiblement dégradée.

Etant donné qu'en Grèce il y a une production importante de fromages à pâte cuite, type Gruyère, dont la technologie de fabrication et les ferments lactiques, mis en œuvre, sont différents de ceux utilisés pour le fromage Feta, nous nous sommes posé la question de savoir si les résultats obtenus, relatifs au fromage Feta, sont valables aussi pour les fromages de type Gruyère.

Pour répondre à cette question nous avons effectué une série de fabrications de fromages, à partir de lait de brebis, d'une part ne contenant pas d'antibiotiques et, d'autre part, à partir de lait contenant différentes concentrations de pénicilline.

\section{MATERIELS ET METHODES}

\section{Le lait}

Il s'agit de lait de brebis, provenant du troupeau de l'école supérieure d'agriculture d'Athènes, qui comprend les races de Chios,

* Laboratoire de Technologie Laitière, Ecole Supérieure d'Agriculture d'Athènes. Votanikos, Athènes (Grèce). 
Frisonne, et des croisements entre celles-ci. Il s'agit d'un mélange de traite, du matin et de l'après-midi, sans antibiotiques.

\section{La présure}

Nous avons employé la présure en poudre Hansen, Danemark, ayant une force de 1/100 000 .

\section{Les ferments}

Les ferments utilisés ont été les ferments lactiques $\mathrm{n}^{\circ} 25 / 30$ et $\mathrm{n}^{\circ} 45$ de la firme Equilait. Le premier est constitué principalement de Strept. lactis et Strept. cremoris, tandis que le second comporte Lact. lactis, Lact. helveticus, Str. lactis et Str. thermophilus.

\section{La pénicilline}

Il s'agit de poudre cristallisée en provenance de U.S.P. "Reference Standards ».

\section{Les analyses}

Le taux d'humidité a été évalué après dessiccation de $5 \mathrm{~g}$ de fromage à $100^{\circ} \mathrm{C}-102^{\circ} \mathrm{C}$, jusqu'à poids constant [7], tandis que le $\mathrm{pH}$ a été mesuré avec un PHmètre Radiometer Copenhague.

\section{La fabrication de fromage}

Après pasteurisation du lait à $63^{\circ} \mathrm{C}$ pendant $30 \mathrm{~min}$ et refroidissement à $36^{\circ} \mathrm{C}$, nous avons ajouté les ferments lactiques $n^{\circ} \quad 25 / 30$ à 1 p. 100 et no 45 à 0,5 p. 100 .

Ensuite, le lait a été séparé en deux parties égales, dans l'une desquelles il y a eu addition de pénicilline, afin d'obtenir la concentration désirée d'antibiotique, tandis que l'autre partie, sans antibiotique, constituait le témoin.

La coagulation s'effectuait à $36^{\circ} \mathrm{C}$ avec $7 \mathrm{~g}$ de présure pour $100 \mathrm{~kg}$ de lait. Ensuite nous avons suivi la technologie mentionnée par Veinoglou et al. [12].

Les fromages fabriqués, deux fromages de $10 \mathrm{~kg}$ par chaque concentration d'antibiotique, ont été examinés du point de vue goût-arôme, texture, couleur et présentation extérieure, Nelson et Trout [9] avec appréciation selon la notation suivante :

\begin{tabular}{llr} 
Goût-arôme & points : $0-45$ \\
Texture & points : $0-35$ \\
Couleur de la pâte & points : $0-10$ \\
Présentation extérieure & points : $0-10$ \\
\cline { 2 - 3 } & total & 100
\end{tabular}




\section{RESULTATS - DISCUSSION}

Ce qui intéresse principalement la fromagerie, c'est l'obtention du meilleur rendement possible à partir d'une quantité donnée de lait et une bonne qualité.

Dans les expériences effectuées, nous avons voulu élucider ces deux données, c'est-à-dire jusqu'à quel point la présence de pénicilline dans le lait de fabrication peut influencer la quantité et la qualité des produits affinés.

Les résultats des expériences, après avoir été analysés statistiquement [5], sont présentés aux tableaux 1 et 2 .

Le tableau 1 montre le rendement fromager pour $100 \mathrm{~kg}$ du lait de brebis employé.

Il est à noter que les essais relatifs à chaque groupe de fromages ont été effectués à des moments différents, ce qui explique les différences remarquées, du point de vue rendement, par rapport à leurs témoins.

Si l'on considère les résultats obtenus à partir du fromage affiné, âgé de $90 \mathrm{j}$, nous ne constatons pas de différences notables entre les fromages et leurs témoins dans les cas de ceux qui étaient fabriqués à partir de lait contenant les quantités de pénicilline de 0,025 - 0,05 0,01 et $0,2 \mathrm{u} . \mathrm{i} / \mathrm{ml}$.

Néanmoins, la différence de rendement en fromage, les 10 premiers jours de maturation, est importante dans le cas du lait qui contenait $0,2 \mathrm{u} . \mathrm{i} / \mathrm{ml}$ de pénicilline.

Cette différence se trouve accentuée le $1^{\text {er }}$ jour de fabrication et elle est statistiquement significative pour $p=0,05$ et par la suite diminue, de telle façon qu'après $10 \mathrm{j}$, bien qu'elle existe toujours, elle se limite au point de n'être pas statistiquement significative. Pour les autres concentrations, il n'a pas été remarqué de telles différences.

Il était évident, et ceci a été confirmé par les analyses relatives, que le haut rendement fromager que nous remarquons dans le cas de l'emploi de lait contenant $0,2 \mathrm{u} . \mathrm{i} / \mathrm{ml}$ de pénicilline, avant $10 \mathrm{j}$ de maturation des fromages, est dû à leur taux élevé d'humidité.

L'antibiotique inhibe les ferments lactiques et, par conséquent, le développement de l'acidité dans les fromages, ayant comme résultat une teneur élevée en humidité.

Le $\mathrm{pH}$ des fromages contenant de la pénicilline est beaucoup plus élevé que celui du témoin et reste ainsi jusqu'à 4 mois ( $\mathrm{pH}$ témoin $4,96-\mathrm{pH}$ fromages + antibiotique 5,12).

Si l'on considère que l'humidité et le $\mathrm{pH}$, durant les différents stades de maturation des fromages, sont des facteurs spécialement importants pour la qualité de ceux-ci, nous devons nous attendre à des différences qualitatives des fromages affinés [4]. 


\section{TABLEAU 1}

Rendement moyen (p. 100/1 de lait de brebis), avec et sans pénicilline, en fromage de type Gruyère

\begin{tabular}{|c|c|c|c|c|c|c|c|}
\hline \multirow{3}{*}{$\begin{array}{l}\text { u.i. de pénicilline/ml } \\
\text { de lait }\end{array}$} & \multicolumn{7}{|c|}{ Rendement p. $100 / 1$} \\
\hline & \multicolumn{7}{|c|}{ Age des fromages (en jours) } \\
\hline & 1 & 3 & 10 & 30 & 40 & 60 & 90 \\
\hline 0,200 & 18,13 & 17,84 & 16,89 & 16,22 & 15,95 & 15,44 & 14,97 \\
\hline 0,000 (témoin) & 17,44 & 17,18 & 16,52 & 15,96 & 15,75 & 15,28 & 14,86 \\
\hline D.S.S. 5 p. $100(P=0,05)$ & $\pm 0,41$ & $\pm 0,35$ & $\pm 0,39$ & - & - & - & $\pm 0,43$ \\
\hline 1 p. $100(\mathrm{P}=0,01)$ & $\pm 0,64$ & $\pm 0,46$ & - & - & - & - & - \\
\hline 0,100 & 18,75 & 15,81 & 17,90 & 17,22 & 16,93 & 16,48 & 15,98 \\
\hline 0,000 (témoin) & 18,67 & 18,42 & 17,88 & 17,26 & 17,00 & 16,59 & 16,12 \\
\hline D.S.S. 5 p. $100(P=0,05)$ & $\pm 0,21$ & - & - & - & - & - & $\pm 0,17$ \\
\hline 0,050 & 18,38 & 18,08 & 17,57 & 16,92 & 16,68 & 16,29 & 15,86 \\
\hline 0,000 (témoin) & 18,34 & 18,04 & 17,55 & 16,99 & 16,70 & 16,39 & 15,87 \\
\hline D.S.S. 5 p. $100(\mathrm{P}=0,05)$ & $\pm 0,27$ & - & - & - & - & - & $\pm 0,09$ \\
\hline 0,025 & 18,16 & 17,82 & 17,30 & 16,62 & 16,42 & 16,01 & 15,55 \\
\hline 0,000 (témoin) & 18,11 & 17,79 & 17,69 & 16,69 & 16,47 & 16,13 & 15,61 \\
\hline D.S.S. 5 p. $100(P=0,05)$ & $\pm 0,28$ & - & - & - & - & - & $\pm 0,24$ \\
\hline
\end{tabular}

D.S.S. = différence statistiquement significative.

Au tableau 2 sont consignés les résultats de l'examen organoleptique de tous les fromages fabriqués. Nous avons constaté qu'une dose de pénicilline de $0,025 \mathrm{u} . \mathrm{i} / \mathrm{ml}$, ajoutée au lait de fabrication, n'avait pas d'influence importante sur la qualité moyenne des fromages fabriqués à partir de celui-ci.

Il faut cependant noter qu'il y a eu des cas, contrairement aux prévisions, où la qualité des fromages contenant de la pénicilline était meilleure que celle des témoins et d'autres où la qualité était inférieure.

Ceci se remarquait lorsque la qualité du témoin était sensiblement en dessous de la qualité moyenne des fromages provenant de lait normal.

Lorsque la quantité de pénicilline augmentait jusqu'à $0,05 \mathrm{u} . \mathrm{i} / \mathrm{ml}$ dans le lait, la qualité des fromages obtenue était assez mauvaise par rapport aux témoins, mais cette différence n'a pas été jugée statistiquement significative. 


\section{TABLEAU 2}

Résultat de l'examen organoleptique du fromage de type Gruyère, fait de lait de brebis, âgé de 4 mois, avec ou sans pénicilline

\begin{tabular}{|c|c|c|c|c|c|}
\hline \multirow{2}{*}{$\begin{array}{l}\text { u.i. de pénicilline } / \mathrm{ml} \\
\text { de lait }\end{array}$} & \multicolumn{5}{|c|}{ Gradation } \\
\hline & $\begin{array}{l}\text { Goût } \\
\text { arôme }\end{array}$ & $\begin{array}{l}\text { Texture } \\
\text { de la } \\
\text { pâte }\end{array}$ & Couleur & $\begin{array}{l}\text { Présenta- } \\
\text { tion } \\
\text { extérieure }\end{array}$ & Total \\
\hline $\begin{array}{l}0,000 \text { (témoin) } \\
0,025 \\
\text { D.S.S. } 5 \text { p. } 100(P=0,05)\end{array}$ & $\begin{array}{r}35,25 \\
36,75 \\
\pm 3,18\end{array}$ & $\begin{array}{r}28,25 \\
29,50 \\
\pm 6,74\end{array}$ & $\begin{array}{r}8,75 \\
9,25 \\
\pm 1,83\end{array}$ & $\begin{array}{r}8,25 \\
8,00 \\
\pm 2,24\end{array}$ & $\begin{array}{r}80,50 \\
82,75 \\
\pm 14,25\end{array}$ \\
\hline $\begin{array}{l}0,000 \text { (témoin) } \\
0,050 \\
\text { D.S.S. } 5 \text { p. } 100(P=0,05)\end{array}$ & $\begin{array}{r}36,83 \\
34,00 \\
\pm 6,76\end{array}$ & $\begin{array}{r}26,17 \\
23,16 \\
\pm 4,55\end{array}$ & $\begin{array}{r}8,33 \\
7,83 \\
\pm 1,28\end{array}$ & $\begin{array}{r}9,66 \\
9,50 \\
\pm 0,28\end{array}$ & $\begin{array}{l}81,00 \\
75,00 \\
\pm 12,90\end{array}$ \\
\hline $\begin{array}{l}0,000 \text { (témoin) } \\
0,100 \\
\text { D.S.S. } 5 \text { p. } 100(P=0,05) \\
\text { 1. p. } 100(P=0,01)\end{array}$ & $\begin{array}{r}38,97 \\
22,66 \\
\pm 6,16 \\
\pm 8,69\end{array}$ & $\begin{array}{r}26,66 \\
21,08 \\
\pm 3,36 \\
\pm 4,74\end{array}$ & $\begin{array}{r}9,48 \\
7,28 \\
\pm 1,82 \\
\pm 2,56\end{array}$ & $\begin{array}{r}9,78 \\
9,20 \\
+0,19 \\
-\end{array}$ & $\begin{array}{r}83,93 \\
60,32 \\
\pm 10,90 \\
\pm 15,39\end{array}$ \\
\hline $\begin{array}{l}0,000 \text { (témoin) } \\
0,200\end{array}$ & $\begin{array}{l}34,25 \\
\text { non né }\end{array}$ & $\begin{array}{r}27,50 \\
\text { ociables }\end{array}$ & - & - & - \\
\hline
\end{tabular}

D.S.S. = différence statistiquement significative.

Il faut cependant noter que pour cette concentration on a noté des cas où la qualité des fromages de lait contenant de la pénicilline était mauvaise quand la qualité des fromages témoins provenant de lait sans antibiotique se trouvait en dessous de la qualité moyenne pour leur catégorie. Dans tous les cas, les fromages fabriqués étaient bons pour être commercialisés.

Par contre, quant au lait de fabrication, il y avait adjonction de 0,10 u.i. de pénicilline/ml la qualité des fromages était sensiblement inférieure à celle des témoins.

Dans tous les cas, il y a eu formation de trous irréguliers avec concentration d'eau dans ceux-ci.

Dans beaucoup de cas, nous avons remarqué une coloration non homogène des fromages ainsi que la formation de taches de couleur claire et de contour indéterminé.

Dans deux cas sur quinze, nous avons noté un goût aigre et une odeur désagréable.

Dans le cas où le lait de fabrication contenait 0,2 u.i. de pénicilline $/ \mathrm{ml}$ les fromages fabriqués ne pouvaient pas être commercialisés. 
Il y avait formation d'un grand nombre de trous anormaux de moyeux blanchâtres et leur texture était gluante.

La figure 1 donne une coupe caractéristique de quatre fromages, où l'on peut remarquer les défauts mentionnés ci-dessus.

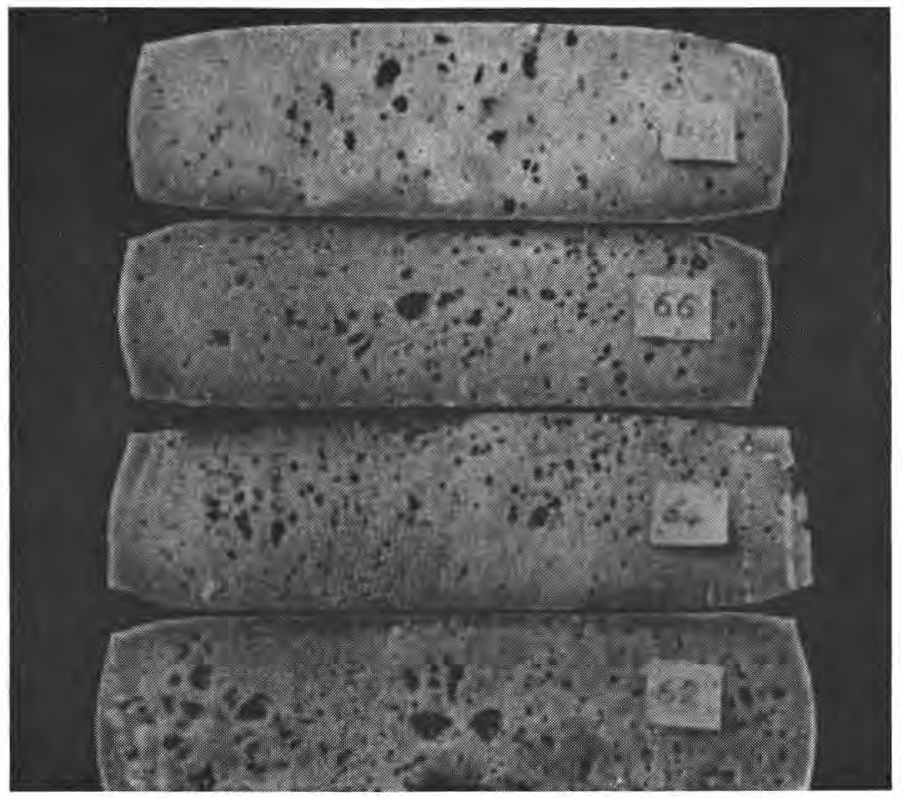

fig. 1

Fromage de type Gruyère fabriqué à partir de lait de brebis avec 0,2 u.i. pénicilline $\mathrm{G} / \mathrm{ml}$.

Il faut noter aussi que dans tous les cas il y a eu gonflement des fromages, plus intense dans le cas des fromages fabriqués à partir de lait contenant des antibiotiques, indépendamment de leur concentration.

Il est évident, d'après les remarques ci-dessus, que le tableau 2 ne donne pas une image complète des résultats obtenus, mais il est dûment complété par les remarques exprimées.

En ce qui concerne l'observation selon laquelle, dans certains cas, avec une concentration de $0,025 \mathrm{u} . \mathrm{i} / \mathrm{ml}$ de lait, en pénicilline contenue dans le lait de fabrication, la qualité des fromages est améliorée, notons que des résultats identiques ont été obtenus par Wessner [13] pour la fabrication de fromages Hergärd quand le lait de fabrication contenait $0,005 \mathrm{u} . \mathrm{i} / \mathrm{ml}$ de pénicilline. 
Il semblerait que la présence de petites quantités d'antibiotique, sous certaines conditions, favorise la dominance de la meilleure microflore pour la maturation.

Suivant Peter et al. [10], la coloration hétérogène des fromages qui contiennent des quantités considérables de pénicilline serait due à la présence de Bac. putrificus, favorisée par le $\mathrm{pH}$ élevé de ces fromages.

En ce qui concerne le goût aigre des fromages, suivant Harwalkar et Seitz [6], il serait dû plutôt à la protéolyse de la caséine qu'à la composition de produits amers provenant de la microflore, tandis que Cjulak [3] et Stadhouders [11] pensent qu'il s'agit d'un mécanisme où il y a action combinée de présure et d'enzymes protéolytiques.

Les peptides qui sont libérés par la présure sont, par la suite, hydrolysés par des souches de streptocoques acido-producteurs qui ne donnent pas d'amertume.

Les micro-organismes formant l'amertume sont considérés comme ne pouvant pas hydrolyser les peptides.

Indépendamment de ces deux hypothèses, il est évident que la présence de pénicilline est la cause de la présence du goût d'amertume.

Comparant les résultats de ce travail avec d'autres concernant le fromage Feta [2], nous constatons que, dans le cas de la Feta, nous avions dégradation de la qualité pour des doses de pénicilline de $0,20 \mathrm{u} . \mathrm{i} / \mathrm{ml}$ et davantage.

Cette différence serait attribuable au fait que pour le cas des fromages de type Gruyère un rôle important serait joué par St. thermophilus et $L$. bulgaricus qui présentent une grande sensibilité à la pénicilline.

\section{CONCLUSION}

Il est évident que, d'après les résultats ci-dessus, les problèmes qui se présentent dans la fabrication du fromage type Gruyère, à partir de lait de brebis contenant de la pénicilline, se trouvent en relation étroite avec la qualité du lait. Il semblerait que, dans le cas des laits de bonne qualité, la présence de pénicilline à une concentration de $0,025 \mathrm{u} . \mathrm{i} / \mathrm{ml}$ n'influence pas défavorablement la qualité du fromage produit.

Par contre, quand la concentration de pénicilline augmente jusqu'à $0,05 \mathrm{u} . \mathrm{i} / \mathrm{ml}$ il y a une dégradation de la qualité à un degré qui dépend de la qualité du lait.

En augmentant la dose de pénicilline jusqu'à $0,10 \mathrm{u.i} / \mathrm{ml}$ la dégradation de la qualité est plus importante, et, à la concentration de $0,2 \mathrm{u} . \mathrm{i} / \mathrm{ml}$, les fromages produits ne peuvent plus être commercialisés. 


\section{Rés u mé}

Le sujet de notre travail a été l'étude de l'influence de quantités croissantes de pénicilline, de 0,025-0,050-0,1 et $0,2 \mathrm{u} . \mathrm{i} / \mathrm{ml}$, sur la qualité d'un fromage de type Gruyère, fabriqué à partir de lait de brebis.

Les fromages fabriqués à partir de lait contenant $0,2 \mathrm{u} . \mathrm{i} / \mathrm{ml}$ de pénicilline, ont présenté un taux d'humidité et un $\mathrm{pH}$ plus élevés que le témoin, spécialement durant les 10 premiers jours de maturation, tandis qu'avec les autres concentrations il n'a pas été constaté de différences notables sur ces points.

L'examen organoleptique que nous avons effectué sur les fromages affinés a montré que la présence d'une quantité de pénicilline de $0,025 \mathrm{u} . \mathrm{i} / \mathrm{ml}$ dans le lait de fabrication, n'influençait pas défavorablement la qualité des fromages. Cependant, nous avons noté aussi, dans certains cas, qu'il y avait une légère amélioration.

Lorsque la concentration de l'antibiotique augmentait, jusqu'à $0,05 \mathrm{u} . \mathrm{i} / \mathrm{ml}$ dans le lait de fabrication, la qualité des fromages se dégradait et ceci était plus intense dans les cas où la qualité des témoins était moins bonne que celle prévue.

Pour les concentrations de pénicilline de $0,1 \mathrm{u} . \mathrm{i} / \mathrm{ml}$ dans le lait de fabrication, l'infériorité de la qualité des fromages était plus remarquable et même, dans deux cas sur quinze, les fromages n'ont pas pu être commercialisés.

\section{S u m m a r y}

\section{EFFECT OF DIFFERENT CONCENTRATIONS OF PENICILLIN IN EWE'S MILK ON THE QUALITY OF GRUYERE TYPE CHEESE}

The effect was examined of different concentrations of penicillin $G$ in sheep's milk on the quality of Gruyère type cheese. It was found that the presence of 0,025 i.u. of penicillin $/ \mathrm{ml}$ in the milk has not any effect on the quality of cheese made from such milk. When concentration of penicillin was increased up to $0,05 \mathrm{i} . \mathrm{u} / \mathrm{ml}$ the cheese produced was of inferior quality. The deterioration was more pronounced when the concentration of the antibiotic was 0,1 i.u/ml. In two cases, out of fifteen, the cheese produced were not negotiable. When the milk contained 0,2 i.u. of penicillin $/ \mathrm{ml}$ all the cheeses produced were not negotiable.

\section{Remerciements}

Nous remercions vivement $\mathrm{M}$. le $\mathrm{Pr} \mathrm{B}$. Veinoglou pour ses précieux conseils. Nous tenons à remercier aussi M. G. Kalatzopoulos et Mme E. Papadopoulou de leurs conseils pour la rédaction de ce travail.

Reçu pour publication en mars 1981. 


\section{Bibliographie}

[1] Albright (J. L.), Tuckey (S. L.) and Woods (G. T.) (1961). - Antibiotics in milk-A review. J. Dairy Sci., 44, 779.

[2] Anifantakis (E. M.) (1980). - Influence de la pénicilline sur la technologie et la qualité du fromage Feta fabriqué à partir du lait de brebis. Le Lait, LX, 598, 525-531.

[3] CuUlaK (J.) (1959). - Bitter flavour in cheese. Aust. J. Dairy Techn., 14, 177.

[4] Davis (J. G.) (1965). - Cheese (Book vol. 1, 163).

[5] Fassoulas (A. K.) (1964). - Elements of experimental Statistic (Book, 37).

[6] Harwalkar (V. R.) and Seitz (E. W.) (1971). - Production of bitter flavor components by lactic cultures. J. Dairy Sci., 54, 12.

[7] International Dairy Federation (1958). - Determination of dry matter in cheese and processed cheese.

[8] Kosikowski (F. V.) and Mocouot (G.) (1958). - Advances in cheese technology, F.A.O., 958, 21.

[9] Nelson (J. G.) and Trout (G. M.). - Judging of dairy products (Book, 233).

[10] Peter (A.), Zollikoffer (E.) and Badaux (F.) (1953). - Manuel de la fabrication du fromage d'Emmental (Book, 110), Edn Hars-Wyss, Berne.

[11] Stadhouders (J.) (1962). - The proteolytic activity of rennet and starter bacteria in cheese with reference to bitter flavour. Int. Dairy Congr., vol. B/353.

[12] Veinoglou (B.), Kalatzopoulos (G.) and Stamelos (N.) (1967). - Contribution to the study of the technology of Gruyère cheese made from sheep's milk. Greek. Agric. Bank, Bul. n ${ }^{\circ} 14$.

[13] Wessner (P.) (1953). - Penicillin in cheese milk and its inactivation with penicillase. Int. Dairy Congr., vol. 3, 1121. 